\title{
Novel treatment strategies for patients with HER2-positive breast cancer who do not benefit from current targeted therapy drugs (Review)
}

\author{
NAN JIANG ${ }^{1,2}$, JING-JING LIN ${ }^{1,2}$, JUN WANG $^{3}$, BEI-NING ZHANG ${ }^{1,4}$, AO LI ${ }^{1,4}$, \\ ZHENG-YANG CHEN ${ }^{1,2}$, SONG GUO ${ }^{1,2}$, BIN-BIN LI ${ }^{1,4}$, YU-ZHONG DUAN $^{1}$, RU-YI YAN ${ }^{4,5}$, \\ HONG-FENG YAN $^{1}$, XIAO-YAN FU ${ }^{1}$, JIN-LIAN ZHOU ${ }^{5}$, HE-MING YANG $^{1}$ and YAN CUI ${ }^{1}$ \\ ${ }^{1}$ Department of General Surgery, 306 Hospital of PLA; ${ }^{2}$ Department of General Surgery, \\ 306 Teaching Hospital of Peking University Health Science Center; ${ }^{3}$ Department of Hepatology, \\ 302 Teaching Hospital of Peking University Health Science Center, Beijing 100101; \\ ${ }^{4}$ Department of General Surgery, PLA 306 Clinical Hospital of Anhui Medical University, Beijing 230000; \\ ${ }^{5}$ Department of Pathology, 306 Hospital of PLA, Beijing 100101, P.R. China
}

Received March 2, 2018; Accepted June 27, 2018

DOI: $10.3892 /$ etm.2018.6459

\begin{abstract}
Human epidermal growth factor receptor-2 positive breast cancer $\left(\mathrm{HER} 2^{+} \mathrm{BC}\right)$ is characterized by a high rate of metastasis and drug resistance. The advent of targeted therapy drugs greatly improves the prognosis of HER $2^{+} \mathrm{BC}$ patients. However, drug resistance or severe side effects have limited the application of targeted therapy drugs. To achieve more effective treatment, considerable research has concentrated on strategies to overcome drug resistance. Abemaciclib (CDK4/6 inhibitor), a new antibody-drug conjugate (ADC), src homology 2 (SH2) containing tyrosine phosphatase-1 (SHP-1)
\end{abstract}

Correspondence to: Dr Yan Cui, Department of General Surgery, 306 Hospital of PLA, An xiang Bei li 9, Beijing 100101, P.R. China E-mail: dryancui@163.com

Abbreviations: ATA, acetyl tanshinone IIA; AE, aloe-emodin; SHP-1, src homology 2 (SH2) containing tyrosine phosphatase-1; IFN- $\gamma$, interferon- $\gamma$; TNF- $\alpha$, tumor necrosis factor- $\alpha$; rVP1, recombinant DNA-derived viral capsid protein-1; EGFR, epidermal growth factor receptor; ER, estrogen receptor; HER2, human epidermal growth factor receptor 2; TK, tyrosine kinase; HP, hesperetin; NG, naringenin; PI3K, phosphatidylinositol 3-kinase; Ras, ras kinase family; Akt, protein kinase B; RAF, receptor activation factor; TSC2, tuberous sclerosis complex 2; MEK, mitogen/extracellular signal-regulated kinase; mTOR, mechanistic target of rapamycin; MAPK, mitogen-activated protein kinase; CCND1, cyclin D1; P70-S6K, ribosomal protein S6 kinase beta-1; S6RP, S6-ribosomal protein; HIF-1 $\alpha$, hypoxia inducible factor $1 \alpha$; YB-1, Y-box binding protein 1; RB, retinoblastoma protein; PD-L1, Programmed death ligand 1; PD-1, programmed death 1; TNBC, triple-negative subtypes of breast cancer

Key words: HER2 target therapy, trastuzumab, neratinib, drug resistance, targeted delivery drugs, natural anti-cancer substances and fatty acid synthase (FASN) have been demonstrated to improve drug resistance. In addition, using an effective vector to accurately deliver drugs to tumors has shown good application prospects. Many studies have also found that natural anti-cancer substances produced effective results during in vitro and in vivo anti-HER2 ${ }^{+} \mathrm{BC}$ research. This review aimed to summarize the current status of potential clinical drugs that may benefit HER2 ${ }^{+} \mathrm{BC}$ patients in the future.

\section{Contents}

1. Introduction

2. Current targeted therapy drugs

3. Does every HER $2^{+}$BC patient benefit from targeted therapy?

4. How should we treat HER2 ${ }^{+} \mathrm{BC}$ patients who do not benefit from trastuzumab, pertuzumab, trastuzumab emtansine (T-DM1) and lapatinib?

5. Conclusions

\section{Introduction}

Breast cancer (BC) is the most diagnosed disease among women and has the second highest incidence among all types of cancer worldwide (1.7 million cases, 11.9\%) (1). Excessive expression of human epidermal growth factor receptor-2 (HER2) has been investigated in $20-30 \%$ of $\mathrm{BC}$ patients and generally recognized as a marker for invasive disease, which is likely to be highly metastatic, to be drug resistant and to spread rapidly (2-4). HER2 is a member of the HER/EGFR/ERBB group of protein kinase superfamily, which receives signals to regulate cell survival, adhesion, motility, proliferation and resistance to apoptosis $(5,6)$. A tyrosine kinase (TK) domain located in the HER2 receptor plays an important role in the occurrence of $\mathrm{BC}$, as it is induced by phosphorylation after 
HER2 homo/hetero dimerization and then activates multiple signal transduction pathways $(5,7)$. The phosphatidylinositol 3-kinase (PI3K) pathway and the Ras/Raf mitogen-activated protein kinase (MAPK) pathway are the most fully investigated of these pathways, both of which may contribute to the acquired resistance against targeted therapy drugs (8-10). Related research has identified that inhibition of the PI3K-Akt pathway with PI3K inhibitors is effective to reverse tumor growth (11-13). When the HER2 proto-oncogene undergoes mutation and conversions into the HER2 oncogene (14), HER2 receptor protein is highly expressed at the cell membrane, and multiple downstream signaling pathways are excessively activated, resulting in uncontrollable cell growth, repeated division of cells, and altered adhesion properties $(7,14,15)$. Based on its unique role in the development of HER2 ${ }^{+} \mathrm{BC}$, HER2 and its downstream signaling pathways have become extremely important targets for therapy. HER $2^{+}$BC that is induced by HER2 amplification was significantly reversed by treatments that targeted HER2 and HER2 TK $(15,16)$. Targeted therapy that depends on the expression levels of HER2 has made great progress, although a considerable portion of the population are resistant.

\section{Current targeted therapy drugs}

Among all of the drugs that target HER 2 and HER2 TK, trastuzumab, pertuzumab, trastuzumab emtansine (T-DM1) and lapatinib have been proven to be effective in treating HER2 ${ }^{+}$ $\mathrm{BC}$ in several clinical trials compared with chemotherapy drugs alone (17-20). Trastuzumab, the first-generation targeted therapy drug, is a humanized monoclonal antibody targeting the extracellular domain of HER-2 $(21,22)$ and has the ability to downregulate the signaling pathways involving PI3K/Akt and MAPK $(23,24)$, which in turn inhibits the proliferation of BC cells that overexpress HER2. Trastuzumab, both administered as a single agent or injected in combination with a series of chemotherapy agents (such as docetaxel or vinorelbine plus trastuzumab (25-27), showed anti-tumor effects and remarkably improved time to progression, response and survival rate $(22,28,29)$. Furthermore, several randomized control trials have revealed that trastuzumab plus chemotherapy drugs significantly reduce the risk of recurrence and death and promote survival incidence compared to chemotherapy drugs alone $(26,27)$, making trastuzumab the cornerstone of adjuvant treatments for HER $2^{+} \mathrm{BC}$.

Pertuzumab belongs to the second-generation targeted drug family, which elicits similar effects to trastuzumab and can significantly promote survival outcomes $(25,30)$. Pertuzumab binds to HER2 and thus blocks the signaling pathways by blocking a binding pocket necessary for receptor dimerization (31). Pertuzumab was approved by FDA as a neoadjuvant therapy in combination with trastuzumab and cytotoxic chemotherapy (32). Related research demonstrated that pertuzumab in combination with trastuzumab was more effective in the blockade of HER2 signaling pathways in vitro and in vivo than either antibody alone (33).

T-DM1, a second-generation antibody, has attracted great interest from researchers for its effective role as an adjuvant and neoadjuvant (34). The phase III EMILIA study and RESA study proved that T-DM1 was efficacious as afunctional treatment and facilitated better prognosis and improvements in health-related quality of life (35-37). T-DM1 has been investigated to promote progression-free survival (PFS) and overall survival (OS) in patients who were HER $2^{+}$and previously treated with trastuzumab and taxane (17).

Lapatinib is a reversible HER2 TK inhibitor that reacts with the ATP binding site, which in turn improves PFS and clinical benefit rate (CBR) by inhibiting the autophosphorylation of ErbB1 and ErbB2 and downstream proliferative signaling pathways $(25,38,39)$. Similarly, in some neoadjuvant clinical trials, lapatinib has been used in dual blockade with trastuzumab to treat patients with HER2 ${ }^{+}$BC $(18,40-42)$.

\section{Does every HER2 ${ }^{+}$BC patient benefit from targeted therapy?}

With the development of targeted therapy, drugs targeting HER 2 ${ }^{+}$BC, such as trastuzumab, pertuzumab and lapatinib, have been proven to significantly improve the prognosis of patients. Though the incidence of PFS, OS and overall response rate $(\mathrm{ORr})$ are significantly promoted among HER2 ${ }^{+} \mathrm{BC}$ patients by treatment with HER2-targeting drugs (17-19), drug resistance and progression of metastatic breast cancer (MBC) still develop gradually and are detrimental to the prognosis of patients. It has been estimated that about half of HER $2^{+}$ MBC does not respond to anti-HER2 drugs (28). Abnormal activation of the PI3K-Akt signaling pathway caused by PI3KCA mutations could promote trastuzumab resistance (43). Similarly, phosphatase and tensin homologue (PTEN) loss also correlated with trastuzumab resistance $(44,45)$. HER2 ${ }^{+}$ BC with PTEN drops and/or PI3K mutations had a worse prognosis $(45,46)$, making this drug resistance a rigorous and persistent clinical challenge. So, patients who present both HER2+ tumors and PI3KCA mutations may benefit from the application of HER2-targeted drugs plus PI3K inhibitors. Rexer et al found that dual HER2 and PI3K blockade in vitro and in xenograft models showed the most effective role in inducing tumor regression, even with a PI3KCA mutation (47). Interestingly, there were also preclinical studies showing that trastuzumab-induced reprogramming of the HER axis resulted in the increase of EGFR and HER3 expression after long-term trastuzumab treatment in cell lines, which may correlate with primary resistance to trastuzumab (48). As mentioned above, trastuzumab resistance in HER $2^{+} \mathrm{BC}$ might be caused by excessive EGFR expression (49,50), making EGFR an effective target to evaluate in response to trastuzumab treatment (51).

In addition to the gradual increase in resistance, another important issue that hampers the application of trastuzumab in clinical patients is side effects, especially cardiomyopathy, which was reported to affect $2.8-3.3 \%$ of patient $(52,53)$. More concerning, when trastuzumab was administered in combination with anthracyclines, cardiac dysfunction, such as left ventricular ejection fraction (LVEF) and congestive heart failure (CHF), affected about a quarter of patients as reported by some studies $(28,54)$.

It has been confirmed that pertuzumab could strengthen the effect of trastuzumab (33). Similarly, a clinical study found that trastuzumab plus pertuzumab received an ORr of $24.2 \%$ and a CBR of 50\% (55). In another clinical trial, a response rate of $18 \%$ was demonstrated after treatment with trastuzumab 
and pertuzumab combination (56); however, over half of the patients did not benefit from treatment.

It is reported that the objective response rate of T-DM1 was approximately $44 \%$ (17), which indicated that more than $50 \%$ of eligible patients did not benefit from T-DM1. In addition, preclinical studies showed that T-DM1 resistance could be caused by downregulation of HER 2 expression $(57,58)$, consistent with decreased activity of T-DM1 in tumors that express low levels of HER2, suggesting that patients who initially responded to T-DM1 might develop acquired resistance as HER2 levels decline. Though a $43.6 \%$ objective response rate was achieved by T-DM1, more than half of patients receive little benefit from treatment (40). At the same time, many clinical trials reported that T-DM1 could bring about side effects, such as fatigue, nausea, thrombocytopenia and headache $(59,60)$.

Lapatinib yielded response rates from $24-53.2 \%$ in clinical activity in advanced HER2-overexpression BC $(17,19,40)$. Although lapatinib was speculated to play a vital role in treating trastuzumab-resistant, HER2-overexpressed tumors, the response rates ranged from 1.4-8.8\% after lapatinib monotherapy $(19,61)$. The addition of lapatinib and capecitabine received a $51 \%$ reduction in the risk of disease progression and a 2-fold time to progression compared to capecitabine alone (16). However, at least half of the patients showed no benefit from treatment.

Furthermore, with the presence of two kinds of drug resistance, 'a primary or inherent resistance and a secondary or acquired resistance' $(24,46,62)$, we are facing two serious challenges: i) Overcoming the drug resistance; and ii) searching for new effective drugs.

\section{How should we treat HER2 ${ }^{+} B C$ patients who do not benefit from trastuzumab, pertuzumab, trastuzumab emtansine (T-DM1) and lapatinib?}

The inherent or acquired resistance to targeted therapy drugs, as well as the relatively serious side effects, limit the application of targeted therapy drugs. These two kinds of unfavorable factors are the driving forces to continue to develop new drugs. To overcome the development of drug resistance, some studies explored the possible treatment methods and identified some substances that had potential value. Specifically, the discovery and application of Taxol greatly improved the prognosis of patients. Interestingly, several studies investigated natural anti-cancer substances that had shown significant anti-cancer effects, which might prevent the tumorigenesis of $\mathrm{BC}$. Furthermore, the new ADC and targeted delivery of drugs were found to be effective in treating HER2 ${ }^{+} \mathrm{BC}$ patients. In this review, we concentrate on some research studies for putative application in clinical treatment. And we summarize the signaling pathways of corresponding effective potential clinical drugs (Fig. 1).

HER2 TK inhibitors. Neratinib is an irreversible kinase inhibitor and a derivative of EKB-569 (EGFR inhibitor) (63-65). Neratinib significantly inhibited EGFR/HER2 kinase after binding to the ATP pocket and blocking downstream signaling pathways (59) and showed anti-cancer bioactivities in HER2-overexpression cell lines and in patients with or without prior trastuzumab treatment (66-68). Neratinib inhibited proliferation and promoted G1-S phase arrest by regulating HER2 and its downstream signaling pathways, specifically through downregulation of pEGFR, pHER2, pAKT, pMEK and $\mathrm{pRb}$ levels and cyclin D1 (CCND1) expression and increase of p27 levels in a HER2-dependent manner (69). Some studies also discovered that neratinib improved trastuzumab resistance and restored sensitivity to trastuzumab in HER2 $2^{+}$BC $(69,70)$. Many clinical trials investigated the functions of neratinib in treating HER $2^{+} \mathrm{BC}$ alone or in combination with trastuzumab, which exhibited great clinical application prospects. A more important role of neratinib was observed when combined with paclitaxel in treating patients who received prior taxane, trastuzumab and lapatinib therapies (71). Neratinib was effective as a single agent or in combination with different chemotherapy drugs in the treatment of HER2+ MBC patients and early BC patients $(68,72-74)$. In a multicenter, randomized, double-blind and placebo-controlled phase III trial (ExteNET), which involved 2,840 women, a total of invasive disease-free survival events of 70 patients in the neratinib group and 109 patients in the placebo group occurred, corresponding to 93.9 and $91.6 \%$ 2-year invasive disease-free survival rates, respectively (75). All of these results above show that neratinib is quite effective and is close to clinical application.

Lapatinib showed significant activities in treating HER2 ${ }^{+}$ $\mathrm{BC}$ either alone or combined with trastuzumab. A considerable portion of HER2 ${ }^{+}$patients were insensitive to lapatinib, which prompted development of a modified drug form. KU004, a derivative of lapatinib, remarkably inhibited pEGFR, pHER2, pAkt and pMAPK in BT474 and NCI-N87 cells, which were treated with lapatinib or not. Treatment with KU004 achieved a G0/G1 phase arrest and corresponding reduction of cells in $\mathrm{S}$ and G2/M phases in BT474 cells. KU004 could induce caspase-dependent apoptosis, as caspase- 8 and caspase- 3 were significantly activated by KU004. In vivo study found that KU004 reduced growth of NCI-N87 xenograft dramatically in a dose-dependent manner by inducing apoptosis. These in vivo and in vitro results indicate that KU004 has the potential to treat $\mathrm{HER} 2^{+} \mathrm{BC}$ in the future (76).

Improvements of drug resistance. Trastuzumab, as the first-generation targeted therapy drug, helped many HER2 ${ }^{+}$ $\mathrm{BC}$ patients to improve their prognosis and quality of life in the future. As we mentioned above, the resistance to trastuzumab limited its application to a large extent. Thus, research and development of new drugs to overcome trastuzumab resistance may contribute significantly to the treatment of HER2+ $\mathrm{BC}$ patients.

It has been proven that CCND1 and CDK4 played a vital role in $\mathrm{BC}(77,78)$. CDK4/6 inhibitors have shown great potential in preclinical studies and clinical trials, such as palbocilcib and ribociclib which has been applied in clinical $(79,80)$. Though CDK4/6 inhibitors are mainly used to treat estrogen receptor $(\mathrm{ER})^{+} / \mathrm{HER} 2^{-}$patients, some studies found that they also have potential therapeutic effect on $\mathrm{ER}^{+} / \mathrm{HER} 2^{+}$patients (81). In an exploratory, open-label, phase 2 study (NA-PHER2), the combination of palbociclib, fulvestrant, trastuzumab and pertuzumab was found reduced the expression of Ki67 significantly (82). Palbocilcib could also efficiently inhibit the proliferation of residual HER $2^{+}$tumor cells that survive T-DM1 in preclinical BC models (83). In 


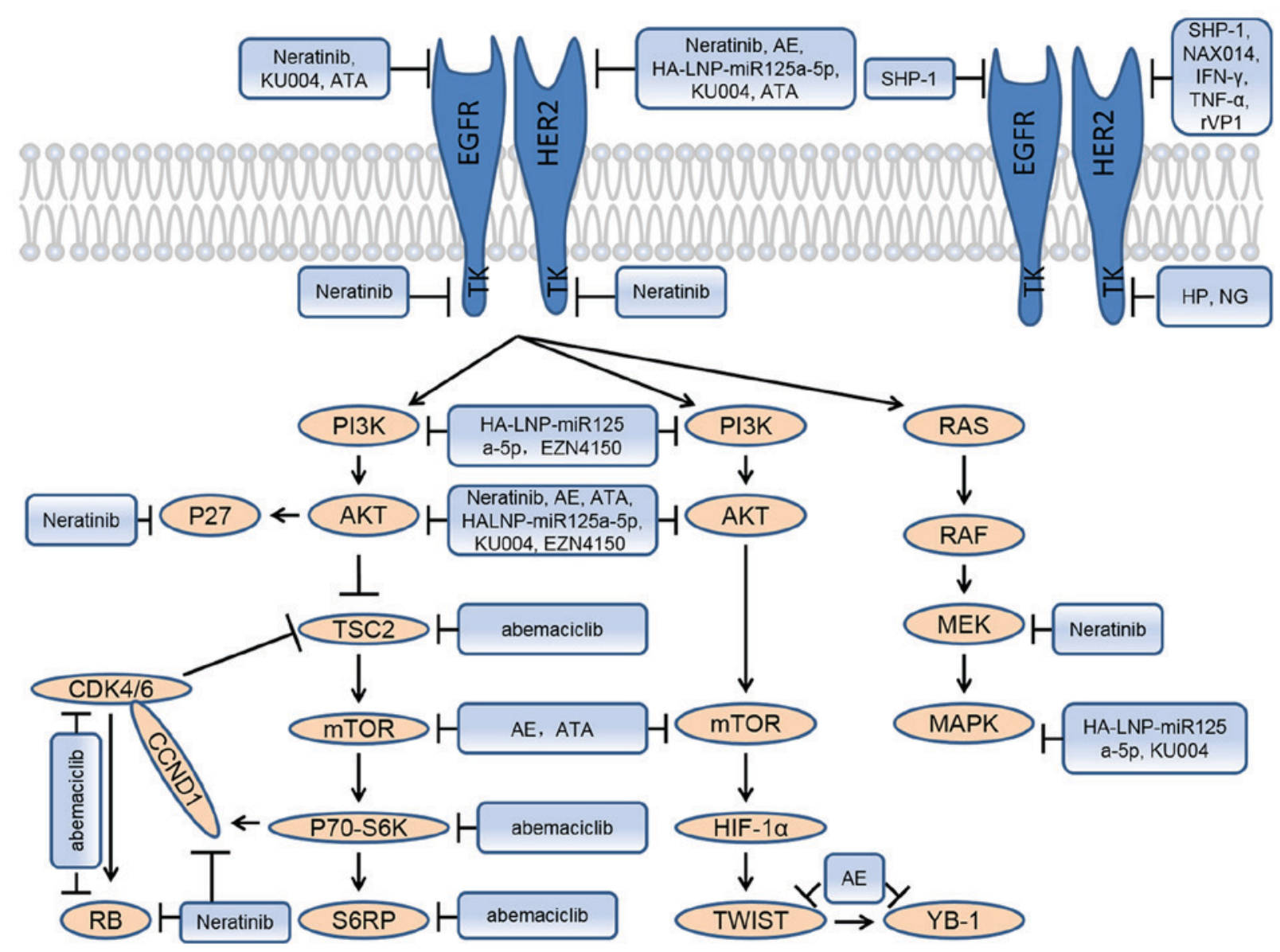

Figure 1. Effectors of EGFR/HER2 downstream signaling pathways and corresponding effective potential clinical drugs. ATA, acetyltanshinone IIA; AE, aloe-emodin; SHP-1, src homology 2 (SH2) containing tyrosine phosphatase-1; IFN- $\gamma$, interferon- $\gamma$; TNF- $\alpha$, tumor necrosis factor- $\alpha$; rVP1, recombinant DNA-derived viral capsid protein-1; EGFR, epidermal growth factor receptor; HER2, human epidermal growth factor receptor 2; TK, tyrosine kinase; HP, hesperetin; NG, naringenin; PI3K, phosphatidylinositol 3-kinase; Ras, ras kinase family; Akt, protein kinase B; RAF, receptor activation factor; TSC2, tuberous sclerosis complex 2; MEK, mitogen/extracellular signal-regulated kinase; mTOR, mechanistic target of rapamycin; MAPK, mitogen-activated protein kinase; CCND1, cyclin D1; P70-S6K, ribosomal protein S6 kinase beta-1; S6RP, S6- ribosomal protein; HIF-1 $\alpha$, hypoxia inducible factor 1 $\alpha$; YB-1, Y-box binding protein $1 ; \mathrm{RB}$, retinoblastoma protein.

a preclinical study, they found that both residual tumor cells and recurrent tumor cells that were present after treatment presented high levels of CCND1and CDK4, which promoted cell proliferation. In addition, abemaciclib, a CDK4/6 inhibitor, inhibited $\mathrm{Rb}$ phosphorylation and significantly postponed the process of recurrent tumors, which showed a CDK4/6-dependent increase in expression of CCND1. Strikingly, reduced sensitivity to both lapatinib and trastuzumab was found when CCND1 was over-expressed in trastuzumab/lapatinib-sensitive BC cell lines. Knockdown of CCND1 in resistant cells partially restored the sensitivity to trastuzumab. Phosphorylation of TSC2 and its downstream effectors, P70-S6K and S6RP, was reduced after abemaciclib treatment, which could be enhanced by combined CDK4/6-HER2 inhibition. Furthermore, in vivo study showed that trastuzumab combined with abemaciclib inhibited tumor growth more than either single agent (84). In another preclinical study, abemaciclib with trastuzumab significantly improved tumor growth inhibition and tumor regressions in xenografts progressing on trastuzumab alone (81). In a phase I study, one patient with hormone receptor-negative and HER2 ${ }^{+}$BC experienced an antitumor effect with a $30 \%$ decrease in tumor size from baseline. At the same time, some clinical trials are ongoing to prove whether CDK4/6 inhibitors could be applied to $\mathrm{ER}^{+} / \mathrm{HER} 2^{+}$patients (NCT02947685; NCT02774681; NCT02907918; NCT02675231) (81).

Antibody-drug conjugates (ADCs) are constructed by covalently attaching small-molecule toxins to antibodies (85-87). T-DM1 is a ADCs that has shown significant activity in treating HER2 ${ }^{+} \mathrm{BC}(86,87)$. A new biparatopic ADC was constructed, consisting of the trastuzumab scFv unit, the 39S Fv unit, and AZ13599185, which inhibits microtubule polymerization during mitosis. The new biparatopic ADC retained HER 2 binding specificity and could effectively deliver cytotoxic agents into the targeted tumors. Compared to T-DM1, the new biparatopic ADC demonstrated at least 10-fold cell killing activity in HER2-expressing cancer cell lines in a HER2-dependent manner and showed activity in cancer cells that were intrinsically resistant to T-DM1. In vivo study suggested the possibility of clinical application of the new biparatopic ADC, as it induced full tumor degradation and inhibited tumor growth in a primary BC xenograft model and an intrinsically or acquired T-DM1-resistant xenograft model, which was not observed after treatment with T- DM1 alone or in combination with pertuzumab. These valuable results show that development of the new biparatopic ADC was necessary 
to explore a more effective drug for the treatment of HER2 ${ }^{+}$ BC (88).

Due to the putative role of EGFR in trastuzumab resistance, blocking EGFR and its corresponding downstream signaling pathways may produce better prognosis. The src homology 2 (SH2) containing tyrosine phosphatase-1 (SHP-1) regulated the intracellular phosphotyrosine level and was reduced or absent in many kinds of cancer cell lines and tissues (89-91). Overexpression of wild-type SHP-1 sensitized trastuzumab-resistant cells to trastuzumab treatment. Furthermore, overexpression of wild-type SHP-1 also significantly upregulated apoptosis and decreased EGFR and HER2, as well as their phosphorylation levels, in trastuzumab-resistant cells. Wild-type SHP-1 could bind to pEGFR and pHER2, and thus reduced both $\mathrm{pEGFR}$ and $\mathrm{pHER} 2$ protein levels. In vivo study demonstrated that SHP-1-overexpression mice showed delayed tumor progression and growth and achieved a better OS after treatment with trastuzumab compared with the control (92).

Fatty acid synthase (FASN) showed activity in promoting tumorigenesis by activating HER2/PI3K/AKT/mTOR and MAPK signaling pathways (93-95). EGFR and pEGFR increased in trastuzumab resistant cells noticeably, which was consistent with the study of Wu et al (92). Pertuzumab combined with EGCG, a classical FASN inhibitor, was more effective than pertuzumab alone against resistant HER2 ${ }^{+}$ $\mathrm{BC}$ cells and increased the apoptosis of cancer cells in a trastuzumab plus lapatinib-resistant HER2+ PDX model compared with pertuzumab alone. In vivo study showed that dual FASN and HER2 blockade achieved more effective tumor reduction compared with EGCG or pertuzumab as single agent (50).

Targeted delivery drugs. The occurrence of all tumors is due to genetic mutations, so gene therapy is the most effective treatment approach for all cancers. Using an effective vector to accurately deliver drugs to the tumors is a very valid treatment method to stop the tumor progression.

A new delivery platform, which was able to escape from lysosomal degradation and distribute symmetrical in the cytoplasm, was established to send mature tumor suppressor microRNA125a-5p to treat HER2 ${ }^{+}$MBC. miR125a-5p significantly decreased cellular proliferation and migration in comparison with control cells, which might have been largely caused by knockdown of the HER 2 mRNA levels. HA-LNP-miR125a-5p reduced the levels of HER2, PI3K, pAKT, Ki67 and pERK1/2 by over 30, 35, 40, 40 and 20\%, respectively, which indicated that HA-LNP-miR 125a-5p could treat $\mathrm{BC}$ through inhibiting HER2 and its downstream signaling pathways (96).

Vaccines that target HER2 ${ }^{+} \mathrm{BC}$ may become the best way to prevent tumorigenesis. Campbell et al (97) had previously shown that the immunostimulatory peptide $\mathrm{Hp} 91$ had potent immune effects, and subsequently, Hp91 was encapsulated inside poly (D,L-lactic-co-glycolic) acid nanoparticles (PLGA-NPs) to construct a possible BC vaccine. The new vaccine significantly increased DCs when compared to free Hp91 in vitro. Furthermore, in vivo study showed that injection of the new vaccine noticeably increased HER2-specific IFN- $\gamma$ spot-forming cells compared to the control group and the free Hp91 group (whether administered at the same concentration or higher). Hp91 encapsulated in PLGA-NPs delayed the tumor development by approximately three months compared to mice that were injected with free Hp91 and delayed the tumor development by approximately 5 months compared to control mice, and it prolonged the OS. These results proved that Hp91 encapsulated inside PLGA-NPs could be developed into an effective $\mathrm{BC}$ vaccine (97).

Natural anti-cancer substances. Natural anti-cancer substances played an important role in the treatment of BC and some new natural anti-cancer substances will play a role in the treatment of HER2 ${ }^{+} \mathrm{BC}$. Paclitaxel is the most successful natural anti-cancer drug, which was extracted first from the bark and wood of Pacific Yew tree (98). Hesperetin (HP) and naringenin (NG) belong to flavonoids, which have shown anti-cancer and pro-apoptotic activity (99). An anthraquinone compound, aloe-emodin (AE), effectively suppressed HER2 expression and cell proliferation in a dose-dependent manner consistent with promotion of apoptosis and G1 cell cycle arrest in HER2 ${ }^{+}$BC cells. Twist and Y-box binding protein 1 (YB-1) have been investigated for promoting cell proliferation, tumor metastasis, invasion, angiogenesis and anticancer-drug resistance $(100,101)$. In vitro study showed that AE observably inhibited cancer cell migration and invasion rates through promoting E-cadherin levels, which restored the epithelial cell adhesion, and inhibiting factors, which activated cancer cell metastasis. These results had important clinical significance and were confirmed by in vivo study in a $\mathrm{SkBr} 3$ cell xenograft model (102). Irisin is newly discovered, secreted from muscle tissue and adipose tissue, and recognized as an adipokine, which has been proven to participate in breast carcinogenesis $(103,104)$. Irisin or its structural analogues may become new therapeutic drugs (105). Several studies have investigated the use of apigenin decreasing the risk of a variety of cancers, including BC (106-108). NAX014, a derivative of berberine (BBR), was effective in inhibiting a variety of cancer cells $(109,110)$ tended to reduce HER2 expression in tumor tissues (111). Acetyl tanshinone IIA (ATA), a tanshinone IIA derivative (112), had shown activity against BC (113). In vivo study showed that injection of ATA reduced tumor volume and tumor weight remarkably (112).

Immune factors. IFN- $\gamma$ and TNF- $\alpha$ had shown antitumor effects (114). This study emphasized the exciting roles of TNF- $\alpha$ and IFN- $\gamma$ in treating HER $2^{+}$BC. No HER $2^{+}$BC cell increase due to induction of cell apoptosis was observed in the dual cytokine-treated group in comparison with the untreated or single cytokine-treated group. To investigate the mechanism of whether TNF- $\alpha$ and IFN- $\gamma$ induced cell apoptosis, they treated TUBO and 4T1 cells with either dual Th1 cytokines, actinomycin $\mathrm{D}$ (positive control), or no treatment (negative control). They found that dual TNF- $\alpha$ and IFN $-\gamma$ treatment decreased pro-caspase-3 levels significantly, corresponding with marked increase in activated caspase- 3 levels. Treatment of Th1 cytokines and selective caspase- 3 agonist (PAC-1) induced HER2 loss observably and showed a strong correlation with cancer cell apoptosis. Interestingly, downregulated HER 2 expression could be eliminated by caspase- $3 / 7$ inhibitor in TUBO cells (115). 
Anti-ErbB2 $\mathrm{mAb}$ therapy failed to reduce tumor growth in IL21R-/- mice; however, anti-ErbB2 mAb therapy succeeded in WT mice, which indicated that the IL21 signaling pathway had a strong correlation with trastuzumab resistance. Furthermore, IL21R expressed by $\mathrm{CD} 8^{+} \mathrm{T}$-cells was required for mice that transferred with WT CD8 ${ }^{+}$T-cells to achieve anti-ErbB2 $\mathrm{mAb}$ therapy success, compared with the mice treated with IL21 $\mathrm{R}^{-1}$ CD8 ${ }^{+}$T-cells. Recombinant IL21 combined with anti-ErbB2 mAb therapy significantly inhibited tumor growth and showed activity in lowering metastatic tumors (116).

Anticancer active substances. Recombinant DNA-derived viral capsid protein-1 (rVP1) has been proven to induce apoptosis and suppress invasion in several cancers. They investigated that both in vivo and in vitro rVP1 treatment significantly inhibited cancer cell metastasis and invasion, consistent with increased E-cadherin and decreased a-vimentin levels in vitro. Furthermore, the mRNA and protein expression of HER 2 were inhibited by rVP1 in a dose-dependent manner (117).

Valproic acid (VPA) showed activity in cell apoptosis (118) and controversial effects in inhibiting BC cells $(119,120)$. During HER2-overexpression in SKBR3 BC cells, cell growth was inhibited by VPA in a dose- and time-dependent manner consistent with observably increased p21 WAF1 expression, which could inhibit tumor cell differentiation and growth $(121,122)$. Cleaved caspase-3 levels were upregulated by $\sim 2$-fold after the treatment with VPA, corresponding with a higher number of TUNEL positive cells in the SKBR3 cell line compared with the control group (123).

EZN4150, an antisense oligonucleotide, is an inhibitor of the PIK3CA gene that encodes the p110 $\alpha$ type I PI3K catalytic subunit. EZN4150 combined with lapatinib or BKM120 (a type I PI3K inhibitor) decreased pAkt and increased cleaved caspase-3 levels, achieving a greater effect in combination than either compound alone. Autophagy is a process that could inhibit tumor initiation but support tumor progression $(124,125)$. The role of autophagy in promoting cell survival was blocked by EZN4150 instead of BKM120 in a p110 $\alpha$-independent manner, which might be mediated by Vps34 ablation, as EZN4150 downregulated both p110 $\alpha$ and Vps34 expression. The combined knockdown of p110 $\alpha$ and Vps34 significantly decreased cell numbers, increased the level of cleaved caspase- 3 and increased lapatinib-mediated growth inhibition in BT474 and SKBR3 cell lines. Although both lapatinib and BYL719 (a p110 $\alpha$-specific PI3K inhibitor (126) treatment robustly induced caspase 3/7 activity, SAR405, an inhibitor of Vps34, more significantly increased caspase 3/7 activity than lapatinib, BYL719 or their combination. These results established Vps34 as a new therapeutic target, and EZN4150 was able to improve clinical prognosis by increasing tumor cell killing (127).

PD-1 and PD-L1 agents. Programmed death ligand 1 (PD-L1) is the ligand of programmed death 1 (PD-1). Anti-PD-1/PD-L1 therapy is a novel immune-checkpoint inhibition therapy and anti-PD-1/PD-L1 agents, such as nivolumab, pembrolizumab, atezolizumab, durvalumab and avelumab have been widely applied to treat various types of cancer (128-130). Anti-PD-1/PD-L1 agents have shown antitumor activity in
$\mathrm{BC}$, especially in the triple-negative subtypes of breast cancer (TNBC) $(131,132)$. But PD-L1 expression is associated with HER $2^{+}$status and there is an independent poor prognostic impact of PD-L1 in HER2 ${ }^{+}$BCs (132-134). In a phase $1 \mathrm{~b}$ trial which 168 patients with $\mathrm{MBC}$ received avelumab, avelumab showed an acceptable clinical activity (135). Currently, many clinical trials in HER $2^{+}$cohort are ongoing, such as NCT02648477 and NCT02129556 for pembrolizumab, NCT02605915 for atezolizumab and NCT02649686 for durvalumab (131). Anti-PD-1/PD-L1 agents will benefit the patients with HER $2^{+} \mathrm{BC}$ in the future.

\section{Conclusions}

The advent of targeted therapy drugs is a revolutionary breakthrough in the history of BC treatment. Trastuzumab is the first-generation targeted therapy drug and has been used widely to treat HER2 ${ }^{+} \mathrm{BC}$. Due to the resistance to trastuzumab and severe side effects, second-generation targeted therapy drugs have been successfully developed and applied for clinical treatment, such as pertuzumab, T-DM1 and lapatinib. Although all of them achieved efficacy in the treatment of HER2 ${ }^{+} \mathrm{BC}$, quite a number of patients did not benefit on account of inherent insensitivity, acquired resistance, or potential side effects. How should we treat this subgroup of HER $2^{+}$BC patients? Elucidating the mechanism of drug resistance and seeking effective drugs to overcome this hurdle may benefit these patients. In addition, new drugs should be developed for clinical application, and many researchers are actively working in this direction, with particular focus on natural anti-cancer substances and accurate delivery drugs that were identified for putative ability to treat HER $2^{+} \mathrm{BC}$. Moreover, these new drugs may also strengthen the effect of targeted therapy drugs in treating HER $2^{+} \mathrm{BC}$ patients who are inherently sensitive to targeted therapy drugs. Based on this review, further investigations are needed to strengthen these findings and identify drugs that can overcome targeted therapy drug-resistance or natural anti-cancer substances that are effective in treating $\mathrm{HER} 2^{+} \mathrm{BC}$.

\section{Acknowledgements}

Not applicable.

\section{Funding}

No funding was received.

\section{Availability of data and materials}

All data generated or analyzed during this study are included in this published article.

\section{Authors' contributions}

YC contributed to the conception of the study. NJ contributed to manuscript preparation and wrote the manuscript. JJL, JW, BNZ, AL, ZYC, SG, BBL, YZD, RYY, HFY, XYF, JLZ and HMY contributed in the writing of the manuscript and assisted in the literature research. 


\section{Ethics approval and consent to participate}

Not applicable.

\section{Patient consent for publication}

Not applicable.

\section{Competing interests}

The authors declare that they have no competing interests.

\section{References}

1. Ferlay J, Soerjomataram I, Dikshit R, Eser S, Mathers C, Rebelo M, Parkin DM, Forman D and Bray F: Cancer incidence and mortality worldwide: Sources, methods and major patterns in GLOBOCAN 2012. Int J Cancer 136: E359-E386, 2015.

2. Hudis CA: Trastuzumab-mechanism of action and use in clinical practice. N Engl J Med 357: 39-51, 2007.

3. Adamczyk A, Niemiec J, Janecka A, Harazin-Lechowska A, Ambicka A, Grela-Wojewoda A, Domagała-Haduch M, Cedrych I, Majchrzyk K, Kruczak A, et al: Original paper prognostic value of PIK3CA mutation status, PTEN and androgen receptor expression for metastasis-free survival in HER2-positive breast cancer patients treated with trastuzumab in adjuvant setting. Pol J Pathol 66: 133-141, 2015.

4. Loibl S and Gianni L: HER2-positive breast cancer. Lancet 389: 2415-2429, 2017.

5. Arteaga CL and Engelman JA: ERBB receptors: From oncogene discovery to basic science to mechanism-based cancer therapeutics. Cancer Cell 25: 282-303, 2014.

6. Petrelli F, Tomasello G, Barni S, Lonati V, Passalacqua R and Ghidini M: Clinical and pathological characterization of HER2 mutations in human breast cancer: A systematic review of the literature. Breast Cancer Res Treat 166: 339-349, 2017.

7. Yarden Y and Sliwkowski MX: Untangling the ErbB signalling network. Nat Rev Mol Cell Biol 2: 127-137, 2001.

8. Wang Q, Liu P, Spangle JM, Von T, Roberts TM, Lin NU, Krop IE, Winer EP and Zhao JJ: PI3K-p110 $\alpha$ mediates resistance to HER2-targeted therapy in HER2+, PTEN-deficient breast cancers. Oncogene 35: 3607-3612, 2016.

9. Ritter CA, Perez-Torres M, Rinehart C, Guix M, Dugger T, Engelman JA and Arteaga CL: Human breast cancer cells selected for resistance to trastuzumab in vivo overexpress epidermal growth factor receptor and ErbB ligands and remain dependent on the ErbB receptor network. Clin Cancer Res 13: 4909-4919, 2007.

10. Larionov AA: Current therapies for human epidermal growth factor receptor 2-positive metastatic breast cancer patients. Front Oncol 8: 89, 2018.

11. Junttila TT, Akita RW, Parsons K, Fields C, Lewis Phillips GD, Friedman LS, Sampath D and Sliwkowski MX Ligand-independent HER2/HER3/PI3K complex is disrupted by trastuzumab and is effectively inhibited by the PI3K inhibitor GDC-0941. Cancer Cell 15: 429-440, 2009.

12. Yakes FM, Chinratanalab W, Ritter CA, King W, Seelig S and Arteaga CL: Herceptin-induced inhibition of phosphatidylinositol-3 kinase and Akt Is required for antibody-mediated effects on p27, cyclin D1, and antitumor action. Cancer Res 62: 4132-4141, 2002.

13. Fry EA, Taneja P and Inoue K: Oncogenic and tumor-suppressive mouse models for breast cancer engaging HER2/neu. Int J Cancer 140: 495-503, 2017.

14. Parsa Y, Mirmalek SA, Kani FE, Aidun A, Salimi-Tabatabaee SA, Yadollah-Damavandi S, Jangholi E, Parsa T and Shahverdi E: A review of the clinical implications of breast cancer biology. Electron Physician 8: 2416-2424, 2016.

15. Nami B and Wang Z: HER2 in breast cancer stemness: A negative feedback loop towards trastuzumab resistance. Cancers (Basel) 9: pii: E40, 2017.

16. Gajria D and Chandarlapaty S: HER2-amplified breast cancer: Mechanisms of trastuzumab resistance and novel targeted therapies. Expert Rev Anticancer Ther 11: 263-275, 2011.
17. Verma S, Miles D, Gianni L, Krop IE, Welslau M, Baselga J, Pegram M, Oh DY, Diéras V, Guardino E, et al: Trastuzumab emtansine for HER2-positive advanced breast cancer. N Engl J Med 367: 1783-1791, 2012.

18. Rimawi MF, Mayer IA, Forero A, Nanda R, Goetz MP, Rodriguez AA, Pavlick AC, Wang T, Hilsenbeck SG, Gutierrez C, et al: Multicenter phase II study of neoadjuvant lapatinib and trastuzumab with hormonal therapy and without chemotherapy in patients with human epidermal growth factor receptor 2-overexpressing breast cancer: TBCRC 006. J Clin Oncol 31: 1726-1731, 2013.

19. Robidoux A, Tang G, Rastogi P, Geyer CE Jr, Azar CA, Atkins JN, Fehrenbacher L, Bear HD, Baez-Diaz L, Sarwar S, et al: Lapatinib as a component of neoadjuvant therapy for HER2-positive operable breast cancer (NSABP protocol B-41): An open-label, randomised phase 3 trial. Lancet Oncol 14: 1183-1192, 2013.

20. Joensuu H: Escalating and de-escalating treatment in HER2-positive early breast cancer. Cancer Treat Rev 52: 1-11, 2017.

21. Ban M, Viculin J, Tomic S, Capkun V, Strikic A, Mise BP, Utrobicic I and Vrdoljak E: Retrospective analysis of efficacy of trastuzumab in adjuvant treatment of HER 2 positive early breast cancer-single institution experience. Neoplasma 63: 761-767, 2016.

22. Cameron D, Piccart-Gebhart MJ, Gelber RD, Procter M, Goldhirsch A, de Azambuja E, Castro G Jr, Untch M, Smith I, Gianni L, et al: 11 years' follow-up of trastuzumab after adjuvant chemotherapy in HER2-positive early breast cancer: Final analysis of the HERceptin Adjuvant (HERA) trial. Lancet 389: 1195-1205, 2017.

23. De P, Hasmann M and Leyland-Jones B: Molecular determinants of trastuzumab efficacy: What is their clinical relevance? Cancer Treat Rev 39: 925-934, 2013.

24. Maximiano S, Magalhães P, Guerreiro MP and Morgado M: Trastuzumab in the treatment of breast cancer. BioDrugs 30: 75-86, 2016.

25. Figueroa-Magalhães MC, Jelovac D, Connolly RM and Wolff AC: Treatment of HER2-positive breast cancer. Breast 23: 128-136, 2014.

26. Zhu ZL, Zhang J, Chen ML and Li K: Efficacy and safety of trastuzumab added to standard treatments for HER2-positive metastatic breast cancer patients. Asian Pac J Cancer Prev 14: 7111-7116, 2013.

27. Osoba D, Slamon DJ, Burchmore M and Murphy M: Effects on quality of life of combined trastuzumab and chemotherapy in women with metastatic breast cancer. J Clin Oncol 20: 3106-3113, 2002.

28. Slamon DJ, Leyland-Jones B, Shak S, Fuchs H, Paton V, Bajamonde A, Fleming T, Eiermann W, Wolter J, Pegram M, et al: Use of chemotherapy plus a monoclonal antibody against HER2 for metastatic breast cancer that overexpresses HER2. N Engl J Med 344: 783-792, 2001.

29. Rugo HS, Barve A, Waller CF, Hernandez-Bronchud M, Herson J, Yuan J, Sharma R, Baczkowski M, Kothekar M, Loganathan S, et al: Effect of a proposed trastuzumab biosimilar compared with trastuzumab on overall response rate in patients with ERBB2 (HER2)-positive metastatic breast cancer: A randomized clinical trial. JAMA 317: 37-47, 2017.

30. Laakmann E, Müller V, Schmidt M and Witzel I: Systemic treatment options for HER2-positive breast cancer patients with brain metastases beyond trastuzumab: A literature review. Breast Care (Basel) 12: 168-171, 2017.

31. Franklin MC, Carey KD, Vajdos FF, Leahy DJ, de Vos AM and Sliwkowski MX: Insights into ErbB signaling from the structure of the ErbB2-pertuzumab complex. Cancer Cell 5: 317-328, 2004.

32. Tiwari SR, Mishra P, Raska P, Calhoun B, Abraham J, Moore H, Budd GT, Fanning A, Valente S, Stewart R, et al: Retrospective study of the efficacy and safety of neoadjuvant docetaxel, carboplatin, trastuzumab/pertuzumab (TCH-P) in nonmetastatic HER2-positive breast cancer. Breast Cancer Res Treat 158: 189-193, 2016.

33. Scheuer W, Friess T, Burtscher H, Bossenmaier B, Endl J and Hasmann M: Strongly enhanced antitumor activity of trastuzumab and pertuzumab combination treatment on HER2-positive human xenograft tumor models. Cancer Res 69: 9330-9336, 2009.

34. Perez EA, Barrios C, Eiermann W, Toi M, Im YH, Conte P, Martin M, Pienkowski T, Pivot X, Burris H III, et al: Trastuzumab emtansine with or without pertuzumab versus trastuzumab plus taxane for human epidermal growth factor receptor 2-positive, advanced breast cancer: Primary results from the phase III MARIANNE study. J Clin Oncol 35: 141-148, 2017. 
35. Cairns L and Curigliano G: Highlights from the 38th SABCS annual meeting, 8th-12th December 2015, San Antonio, USA. Ecancermedicalscience 10: 618, 2016.

36. Welslau M, Diéras V, Sohn JH, Hurvitz SA, Lalla D, Fang L, Althaus B, Guardino E and Miles D: Patient-reported outcomes from EMILIA, a randomized phase 3 study of trastuzumab emtansine (T-DM1) versus capecitabine and lapatinib in human epidermal growth factor receptor 2-positive locally advanced or metastatic breast cancer. Cancer 120: 642-651, 2014.

37. Krop IE, Kim SB, Martin AG, LoRusso PM, Ferrero JM, Badovinac-Crnjevic T, Hoersch S, Smitt $M$ and Wildiers H: Trastuzumab emtansine versus treatment of physician's choice in patients with previously treated HER2-positive metastatic breas cancer(TH3RESA): Final overall survival results from a randomised open-label phase 3 trial. Lancet Oncol 18: 743-754, 2017.

38. Blackwell KL, Burstein HJ, Storniolo AM, Rugo H, Sledge G, Koehler M, Ellis C, Casey M, Vukelja S, Bischoff J, et al: Randomized study of Lapatinib alone or in combination with trastuzumab in women with ErbB2-positive, trastuzumab-refractory metastatic breast cancer. J Clin Oncol 28: 1124-1130, 2010.

39. Spector NL, Xia W, Burris H III, Hurwitz H, Dees EC, Dowlati A O'Neil B, Overmoyer B, Marcom PK, Blackwell KL, et al: Study of the biologic effects of lapatinib, a reversible inhibitor of ErbB1 and ErbB2 tyrosine kinases, on tumor growth and survival pathways in patients with advanced malignancies. J Clin Oncol 23: 2502-2512, 2005.

40. Guarneri V, Frassoldati A, Bottini A, Cagossi K, Bisagni G, Sarti S, Ravaioli A, Cavanna L, Giardina G, Musolino A, et al: Preoperative chemotherapy plus trastuzumab, lapatinib, or both in human epidermal growth factor receptor 2-positive operable breast cancer: Results of the randomized phase II CHER-LOB study. J Clin Oncol 30: 1989-1995, 2012.

41. Xu ZQ, Zhang Y, Li N, Liu PJ, Gao L, Gao X and Tie XJ: Efficacy and safety of lapatinib and trastuzumab for HER2-positive breast cancer: A systematic review and meta-analysis of randomised controlled trials. BMJ Open 7: e013053, 2017.

42. Solinas C, Ceppi M, Lambertini M, Scartozzi M, Buisseret L, Garaud S, Fumagalli D, de Azambuja E, Salgado R, Sotiriou C, et al: Tumor-infiltrating lymphocytes in patients with HER2-positive breast cancer treated with neoadjuvant chemotherapy plus trastuzumab, lapatinib or their combination: A meta-analysis of randomized controlled trials. Cancer Treat Rev 57: 8-15, 2017.

43. Hanker AB, Pfefferle AD, Balko JM, Kuba MG, Young CD Sánchez V, Sutton CR, Cheng H, Perou CM, Zhao JJ, et al: Mutant PIK3CA accelerates HER2-driven transgenic mammary tumors and induces resistance to combinations of anti-HER2 therapies. Proc Natl Acad Sci USA 110: 14372-14377, 2013.

44. Nagata Y, Lan KH, Zhou X, Tan M, Esteva FJ, Sahin AA, Klos KS, Li P, Monia BP, Nguyen NT, et al: PTEN activation contributes to tumor inhibition by trastuzumab, and loss of PTEN predicts trastuzumab resistance in patients. Cancer Cell 6: $117-127,2004$

45. Singh JC, Jhaveri K and Esteva FJ: HER2-positive advanced breast cancer: Optimizing patient outcomes and opportunities for drug development. Br J Cancer 111: 1888-1898, 2014.

46. Vu T and Claret FX: Trastuzumab: Updated mechanisms of action and resistance in breast cancer. Front Oncol 2: 62, 2012.

47. Rexer BN, Chanthaphaychith S, Dahlman K and Arteaga CL: Direct inhibition of PI3K in combination with dual HER 2 inhibitors is required for optimal antitumor activity in HER2+ breast cancer cells. Breast Cancer Res 16: R9, 2014.

48. Narayan M, Wilken JA, Harris LN, Baron AT, Kimbler KD and Maihle NJ: Trastuzumab-induced HER reprogramming in 'resistant' breast carcinoma cells. Cancer Res 69: 2191-2194, 2009

49. Diermeier S, Horváth G, Knuechel-Clarke R, Hofstaedter F, Szöllosi J and Brockhoff G: Epidermal growth factor receptor coexpression modulates susceptibility to Herceptin in HER2/neu overexpressing breast cancer cells via specific erbB-receptor interaction and activation. Exp Cell Res 304: 604-619, 2005.

50. Blancafort A, Giró-Perafita A, Oliveras G, Palomeras S, Turrado C, Campuzano Ò, Carrión-Salip D, Massaguer A Brugada R, Palafox M, et al: Dual fatty acid synthase and HER2 signaling blockade shows marked antitumor activity against breast cancer models resistant to anti-HER 2 drugs. PLoS One 10 e0131241, 2015.

51. O'Donovan N, Byrne AT, O'Connor AE, McGee S, Gallagher WM and Crown J: Synergistic interaction between trastuzumab and EGFR/HER-2 tyrosine kinase inhibitors in HER-2 positive breast cancer cells. Invest New Drugs 29: 752-759, 2011.
52. Perez EA, Suman VJ, Davidson NE, Sledge GW, Kaufman PA, Hudis CA, Martino S, Gralow JR, Dakhil SR, Ingle JN, et al: Cardiac safety analysis of doxorubicin and cyclophosphamide followed by paclitaxel with or without trastuzumab in the North central cancer treatment group N9831 adjuvant breast cancer trial. J Clin Oncol 26: 1231-1238, 2008.

53. Dokmanovic M, King KE, Mohan N, Endo Y and Wu WJ: Cardiotoxicity of ErbB2-targeted therapies and its impact on drug development, a spotlight on trastuzumab. Expert Opin Drug Metab Toxicol 13: 755-766, 2017.

54. Keefe DL: Trastuzumab-associated cardiotoxicity. Cancer 95: 1592-1600, 2002

55. Baselga J, Gelmon KA, Verma S, Wardley A, Conte P, Miles D, Bianchi G, Cortes J, McNally VA, Ross GA, et al: Phase II trial of pertuzumab and trastuzumab in patients with human epidermal growth factor receptor 2-positive metastatic breast cancer that progressed during prior trastuzumab therapy. J Clin Oncol 28: $1138-1144,2010$.

56. Portera CC, Walshe JM, Rosing DR, Denduluri N, Berman AW, Vatas U, Velarde M, Chow CK, Steinberg SM, Nguyen D, et al: Cardiac toxicity and efficacy of trastuzumab combined with pertuzumab in patients with [corrected] human epidermal growth factor receptor 2-positive metastatic breast cancer. Clin Cancer Res 14: 2710-2716, 2008.

57. Burris HA III, Rugo HS, Vukelja SJ, Vogel CL, Borson RA, Limentani S, Tan-Chiu E, Krop IE, Michaelson RA, Girish S, et al: Phase II study of the antibody drug conjugate trastuzumab-DM1 for the treatment of human epidermal growth factor receptor 2 (HER2)-positive breast cancer after prior HER2-directed therapy. J Clin Oncol 29: 398-405, 2011.

58. LoRusso PM, Weiss D, Guardino E, Girish S and Sliwkowski MX: Trastuzumab emtansine: A unique antibody-drug conjugate in development for human epidermal growth factor receptor 2-positive cancer. Clin Cancer Res 17: 6437-6447, 2011.

59. Kowalczyk L, Bartsch R, Singer CF and Farr A: Adverse events of trastuzumab emtansine (T-DM1) in the treatment of HER2-positive breast cancer patients. Breast Care (Basel) 12: 401-408, 2017.

60. Watanabe J, Ito Y, Saeki T, Masuda N, Takano T, Takao S, Nakagami K, Tsugawa K, Nakagawa S, Kanatani K and Nakayama T: Safety evaluation of trastuzumab emtansine in japanese patients with HER2-positive advanced breast cancer. In Vivo 31: 493-500, 2017

61. Blackwell KL, Pegram MD, Tan-Chiu E, Schwartzberg LS, Arbushites MC, Maltzman JD, Forster JK, Rubin SD, Stein SH and Burstein HJ: Single-agent lapatinib for HER2-overexpressing advanced or metastatic breast cancer that progressed on first- or second-line trastuzumab-containing regimens. Ann Oncol 20: 1026-1031, 2009

62. Ahmad S, Gupta S, Kumar R, Varshney GC and Raghava GP: Herceptin resistance database for understanding mechanism of resistance in breast cancer patients. Sci Rep 4: 4483, 2014.

63. Rabindran SK, Discafani CM, Rosfjord EC, Baxter M, Floyd MB, Golas J, Hallett WA, Johnson BD, Nilakantan R, Overbeek E, et al: Antitumor activity of HKI-272, an orally active, irreversible inhibitor of the HER-2 tyrosine kinase. Cancer Res 64: 3958-3965, 2004.

64. Tsou HR, Overbeek-Klumpers EG, Hallett WA, Reich MF, Floyd MB, Johnson BD, Michalak RS, Nilakantan R, Discafani C, Golas J, et al: Optimization of 6,7-disubstituted-4-(arylamino) quinoline-3-carbonitriles as orally active, irreversible inhibitors of human epidermal growth factor receptor-2 kinase activity. J Med Chem 48: 1107-1131, 2005.

65. Keyvanjah K, DiPrimeo D, Li A, Obaidi M, Swearingen D and Wong A: Pharmacokinetics of neratinib during coadministration with lansoprazole in healthy subjects. Br J Clin Pharmacol 83: 554-561, 2017.

66. Saura C, Garcia-Saenz JA, Xu B, Harb W, Moroose R, Pluard T, Cortés J, Kiger C, Germa C, Wang K, et al: Safety and efficacy of neratinib in combination with capecitabine in patients with metastatic human epidermal growth factor receptor 2-positive breast cancer. J Clin Oncol 32: 3626-3633, 2014.

67. Burstein HJ, Sun Y, Dirix LY, Jiang Z, Paridaens R, Tan AR, Awada A, Ranade A, Jiao S, Schwartz G, et al: Neratinib, an irreversible ErbB receptor tyrosine kinase inhibitor, in patients with advanced ErbB2-positive breast cancer. J Clin Oncol 28: 1301-1307, 2010.

68. Echavarria I, López-Tarruella S, Márquez-Rodas I, Jerez Y and Martin M: Neratinib for the treatment of HER2-positive early stage breast cancer. Expert Rev Anticancer Ther 17: 669-679, 2017. 
69. Canonici A, Gijsen M, Mullooly M, Bennett R, Bouguern N Pedersen K, O'Brien NA, Roxanis I, Li JL, Bridge E, et al: Neratinib overcomes trastuzumab resistance in HER2 amplified breast cancer. Oncotarget 4: 1592-1605, 2013.

70. Mohd Nafi SN, Generali D, Kramer-Marek G, Gijsen M, Strina C Cappelletti M, Andreis D, Haider S, Li JL, Bridges E, et al: Nuclear HER4 mediates acquired resistance to trastuzumab and is associated with poor outcome in HER 2 positive breast cancer. Oncotarget 5: 5934-5949, 2014.

71. Jankowitz RC, Abraham J, Tan AR, Limentani SA, Tierno MB, Adamson LM, Buyse M, Wolmark N and Jacobs SA: Safety and efficacy of neratinib in combination with weekly paclitaxel and trastuzumab in women with metastatic HER2-positive breast cancer: An NSABP foundation research program phase I study. Cancer Chemother Pharmacol 72: 1205-1212,2013.

72. Kourie HR, Chaix M, Gombos A, Aftimos P and Awada A: Pharmacodynamics, pharmacokinetics and clinical efficacy of neratinib in HER2-positive breast cancer and breast cancer with HER2 mutations. Expert Opin Drug Metab Toxicol 12: 947-957, 2016

73. Chan A: Neratinib in HER-2-positive breast cancer: Results to date and clinical usefulness. Ther Adv Med Oncol 8: 339-350, 2016

74. Kourie HR, El Rassy E, Clatot F, de Azambuja E and Lambertini M: Emerging treatments for HER2-positive early-stage breast cancer: Focus on neratinib. Onco Targets Ther 10: 3363-3372, 2017.

75. Chan A, Delaloge S, Holmes FA, Moy B, Iwata H, Harvey VJ, Robert NJ, Silovski T, Gokmen E, von Minckwitz G, et al: Neratinib after trastuzumab-based adjuvant therapy in patients with HER2-positive breast cancer (ExteNET): A multicentre, randomised, double-blind, placebo-controlled, phase 3 trial. Lancet Oncol 17: 367-377, 2016

76. Tian C, Ding P, Yuan Z, Li H, Zhao Y, Sun L, Guo Q, Wang Z, Sun L, Zhang L and Jiang Z: A novel dual EGFR/HER2 inhibitor KU004 induces cell cycle arrest and apoptosis in HER2-overexpressing cancer cells. Apoptosis 20: 1599-1612, 2015

77. Choi YJ, Li X, Hydbring P, Sanda T, Stefano J, Christie AL, Signoretti S, Look AT, Kung AL, von Boehmer H and Sicinski P: The requirement for cyclin $\mathrm{D}$ function in tumor maintenance. Cancer Cell 22: 438-451, 2012.

78. Yu Q, Geng Y and Sicinski P: Specific protection against breast cancers by cyclin D1 ablation. Nature 411: 1017-1021, 2001.

79. Iwata $\mathrm{H}$ : Clinical development of CDK4/6 inhibitor for breast cancer. Breast Cancer 25: 402-406, 2018

80. Walker AJ, Wedam S, Amiri-Kordestani L, Bloomquist E, Tang S, Sridhara R, Chen W, Palmby TR, Fourie Zirkelbach J, $\mathrm{Fu}$ W, et al: FDA approval of palbociclib in combination with fulvestrant for the treatment of hormone receptor-positive, HER2-negative metastatic breast cancer. Clin Cancer Res 22: 4968-4972, 2016.

81. Kwapisz D: Cyclin-dependent kinase 4/6 inhibitors in breast cancer: Palbociclib, ribociclib, and abemaciclib. Breast Cancer Res Treat 166: 41-54, 2017

82. Gianni L, Bisagni G, Colleoni M, Del Mastro L, Zamagni C, Mansutti M, Zambetti M, Frassoldati A, De Fato R, Valagussa P and Viale G: Neoadjuvant treatment with trastuzumab and pertuzumab plus palbociclib and fulvestrant in HER2-positive, ER-positive breast cancer (NA-PHER2): An exploratory, open-label, phase 2 study. Lancet Oncol 19: 249-256, 2018

83. Witkiewicz AK, Cox D and Knudsen ES: CDK4/6 inhibition provides a potent adjunct to Her2-targeted therapies in preclinical breast cancer models. Genes Cancer 5: 261-272, 2014.

84. Goel S, Wang Q, Watt AC, Tolaney SM, Dillon DA, Li W, Ramm S, Palmer AC, Yuzugullu H, Varadan V, et al: Overcoming therapeutic resistance in HER2-positive breas cancers with CDK4/6 inhibitors. Cancer Cell 29: 255-269, 2016.

85. Sievers EL and Senter PD: Antibody-drug conjugates in cancer therapy. Annu Rev Med 64: 15-29, 2013

86. Lianos GD, Vlachos K, Zoras O, Katsios C, Cho WC and Roukos DH: Potential of antibody-drug conjugates and nove therapeutics in breast cancer management. Onco Targets Ther 7: 491-500, 2014

87. Padayachee ER, Biteghe FAN, Malindi Z, Bauerschlag D and Barth S: Human antibody fusion proteins/antibody drug conjugates in breast and ovarian cancer. Transfus Med Hemother 44: 303-310, 2017
88. Li JY, Perry SR, Muniz-Medina V, Wang X, Wetzel LK, Rebelatto MC, Hinrichs MJ, Bezabeh BZ, Fleming RL, Dimasi N, et al: A biparatopic HER2-targeting antibody-drug conjugate induces tumor regression in primary models refractory to or ineligible for HER2-targeted therapy. Cancer Cell 29: 117-129, 2016.

89. Tsui FW, Martin A, Wang J and Tsui HW: Investigations into the regulation and function of the $\mathrm{SH} 2$ domain-containing protein-tyrosine phosphatase, SHP-1. Immunol Res 35: 127-136, 2006.

90. Liu CY, Chen KF, Chao TI, Chu PY, Huang CT, Huang TT, Yang HP, Wang WL, Lee CH, Lau KY, et al: Sequential combination of docetaxel with a SHP-1 agonist enhanced suppression of p-STAT3 signaling and apoptosis in triple negative breast cancer cells. J Mol Med (Berl) 95: 965-975, 2017.

91. Liu CY, Tseng LM, Su JC, Chang KC, Chu PY, Tai WT, Shiau CW and Chen KF: Erratum to: Novel sorafenib analogues induce apoptosis through SHP-1 dependent STAT3 inactivation in human breast cancer cells. Breast Cancer Res 19: 5, 2017.

92. Wu Y, Li R, Zhang J, Wang G, Liu B, Huang X, Zhang T and Luo R: Protein tyrosine phosphatase SHP-1 sensitizes EGFR/HER-2 positive breast cancer cells to trastuzumab through modulating phosphorylation of EGFR and HER-2. Onco Targets Ther 8: 2577-2587, 2015.

93. Puig T, Turrado C, Benhamú B, Aguilar H, Relat J, Ortega-Gutiérrez S, Casals G, Marrero PF, Urruticoechea A, Haro D, et al: Novel inhibitors of fatty acid synthase with anticancer activity. Clin Cancer Res 15: 7608-7615, 2009.

94. Grunt TW, Wagner R, Grusch M, Berger W, Singer CF, Marian B, Zielinski CC and Lupu R: Interaction between fatty acid synthase- and ErbB-systems in ovarian cancer cells. Biochem Biophys Res Commun 385: 454-459, 2009.

95. Menendez JA and Lupu R: Fatty acid synthase (FASN) as a therapeutic target in breast cancer. Expert Opin Ther Targets 21: 1001-1016, 2017

96. Hayward SL, Francis DM, Kholmatov P and Kidambi S: Targeted delivery of MicroRNA125a-5p by engineered lipid nanoparticles for the treatment of HER2 positive metastatic breast cancer. J Biomed Nanotechnol 12: 554-568, 2016.

97. Campbell DF, Saenz R, Bharati IS, Seible D, Zhang L, Esener S, Messmer B, Larsson M and Messmer D: Enhanced anti-tumor immune responses and delay of tumor development in human epidermal growth factor receptor 2 mice immunized with an immunostimulatory peptide in poly(D,L-lactic-co-glycolic) acid nanoparticles. Breast Cancer Res 17: 48, 2015.

98. Li J, Wang F, Sun D and Wang R: A review of the ligands and related targeting strategies for active targeting of paclitaxel to tumours. J Drug Target 24: 590-602, 2016.

99. Chandrika BB, Steephan M, Kumar TR, Sabu A and Haridas M: Hesperetin and naringenin sensitize HER 2 positive cancer cells to death by serving as HER2 tyrosine kinase inhibitors. Life Sci 160: 47-56, 2016.

100. Nishio S, Ushijima K, Yamaguchi T, Sasajima Y, Tsuda H, Kasamatsu T, Kage M, Ono M, Kuwano M and Kamura T: Nuclear Y-box-binding protein-1 is a poor prognostic marker and related to epidermal growth factor receptor in uterine cervical cancer. Gynecol Oncol 132: 703-708, 2014.

101. He L, Che M, Hu J, Li S, Jia Z, Lou W, Li C, Yang J, Sun S, Wang $\mathrm{H}$ and Chen $\mathrm{X}$ : Twist contributes to proliferation and epithelial-to-mesenchymal transition-induced fibrosis by regulating YB-1 in human peritoneal mesothelial cells. Am J Pathol 185: 2181-2193, 2015.

102. Ma JW, Hung CM, Lin YC, Ho CT, Kao JY and Way TD Aloe-emodin inhibits HER-2 expression through the downregulation of Y-box binding protein-1 in HER-2-overexpressing human breast cancer cells. Oncotarget 7: 58915-58930, 2016.

103. Housa D, Housová J, VernerováZ and Haluzík M: Adipocytokines and cancer. Physiol Res 55: 233-244, 2006.

104. Vona-Davis L and Rose DP: Adipokines as endocrine, paracrine, and autocrine factors in breast cancer risk and progression. Endocr Relat Cancer 14: 189-206, 2007.

105. Provatopoulou X, Georgiou GP, Kalogera E, Kalles V, Matiatou MA, Papapanagiotou I, Sagkriotis A, Zografos GC and Gounaris A: Serum irisin levels are lower in patients with breast cancer: Association with disease diagnosis and tumor characteristics. BMC Cancer 15: 898, 2015.

106. Shukla S, Bhaskaran N, Babcook MA, Fu P, Maclennan GT and Gupta S: Apigenin inhibits prostate cancer progression in TRAMP mice via targeting PI3K/Akt/FoxO pathway. Carcinogenesis 35: 452-460, 2014. 
107. Seo HS, Ju JH, Jang K and Shin I: Induction of apoptotic cell death by phytoestrogens by up-regulating the levels of phospho-p53 and p21 in normal and malignant estrogen receptor $\alpha$-negative breast cells. Nutr Res 31: 139-146, 2011.

108. Seo HS, Jo JK, Ku JM, Choi HS, Choi YK, Woo JK, Kim HI, Kang SY, Lee KM, Nam KW, et al: Induction of caspase-dependent extrinsic apoptosis by apigenin through inhibition of signal transducer and activator of transcription 3 (STAT3) signalling in HER2-overexpressing BT-474 breast cancer cells. Biosci Rep 35: pii: e00276, 2015.

109. Liu Q, Xu X, Zhao M, Wei Z, Li X, Zhang X, Liu Z, Gong Y and Shao C: Berberine induces senescence of human glioblastoma cells by downregulating the EGFR-MEK-ERK signaling pathway. Mol Cancer Ther 14: 355-363, 2015.

110. Chu SC, Yu CC, Hsu LS, Chen KS, Su MY and Chen PN: Berberine reverses epithelial-to-mesenchymal transition and inhibits metastasis and tumor-induced angiogenesis in human cervical cancer cells. Mol Pharmacol 86: 609-623, 2014.

111. Pierpaoli E, Damiani E, Orlando F, Lucarini G, Bartozzi B, Lombardi P, Salvatore C, Geroni C, Donati A and Provinciali M: Antiangiogenic and antitumor activities of berberine derivative NAX014 compound in a transgenic murine model of HER2/neu-positive mammary carcinoma. Carcinogenesis 36 1169-1179, 2015.

112. Guerram M, Jiang ZZ, Yousef BA, Hamdi AM, Hassan HM, Yuan ZQ, Luo HW, Zhu X and Zhang LY: The potential utility of acetyltanshinone IIA in the treatment of HER2-overexpressed breast cancer: Induction of cancer cell death by targeting apoptotic and metabolic signaling pathways. Oncotarget 6 : 21865-21877, 2015.

113. Tian HL, Yu T, Xu NN, Feng C, Zhou LY, Luo HW, Chang DC, Le XF and Luo KQ: A novel compound modified from tanshinone inhibits tumor growth in vivo via activation of the intrinsic apoptotic pathway. Cancer Lett 297: 18-30, 2010.

114. Guan YQ, Li Z and Liu JM: Death signal transduction induced by co-immobilized TNF- $\alpha$ plus IFN- $\gamma$ and the development of polymeric anti-cancer drugs. Biomaterials 31: 9074-9085, 2010.

115. Namjoshi P, Showalter L, Czerniecki BJ and Koski GK: T-helper 1-type cytokines induce apoptosis and loss of HER-family oncodriver expression in murine and human breast cancer cells. Oncotarget: doi.org/10.18632/oncotarget.10298.

116. Mittal D, Caramia F, Michiels S, Joensuu H, Kellokumpu-Lehtinen PL, Sotiriou C, Loi S and Smyth MJ: Improved treatment of breast cancer with anti-HER2 therapy requires interleukin-21 signaling in $\mathrm{CD} 8+\mathrm{T}$ cells. Cancer Res 76: 264-274, 2016.

117. Hung SW, Chiu CF, Chen TA, Chu CL, Huang CC, Shyur LF, Liang CM and Liang SM: Recombinant viral protein VP1 suppresses HER-2 expression and migration/metastasis of breast cancer. Breast Cancer Res Treat 136: 89-105, 2012.

118. Takai $\mathrm{N}$ and Narahara H: Human endometrial and ovarian cancer cells: Histone deacetylase inhibitors exhibit antiproliferative activity, potently induce cell cycle arrest, and stimulate apoptosis. Curr Med Chem 14: 2548-2553, 2007.

119. Travaglini L, Vian L, Billi M, Grignani F and Nervi C: Epigenetic reprogramming of breast cancer cells by valproic acid occurs regardless of estrogen receptor status. Int J Biochem Cell Biol 41: 225-234, 2009.

120.Zhang L, Wang G, Wang L, Song C, Leng Y, Wang X and Kang J: VPA inhibits breast cancer cell migration by specifically targeting HDAC2 and down-regulating Survivin. Mol Cell Biochem 361: 39-45, 2012.
121. Hrzenjak A, Moinfar F, Kremser ML, Strohmeier B, Staber PB, Zatloukal $\mathrm{K}$ and Denk H: Valproate inhibition of histone deacetylase 2 affects differentiation and decreases proliferation of endometrial stromal sarcoma cells. Mol Cancer Ther 5: 2203-2210, 2006.

122. Rocchi P, Tonelli R, Camerin C, Purgato S, Fronza R, Bianucci F, Guerra F, Pession A and Ferreri AM: p21Waf1/Cip1 is a common target induced by short-chain fatty acid HDAC inhibitors (valproic acid, tributyrin and sodium butyrate) in neuroblastoma cells. Oncol Rep 13: 1139-1144, 2005.

123. Mawatari T, Ninomiya I, Inokuchi M, Harada S, Hayashi $H$, Oyama K, Makino I, Nakagawara H, Miyashita T, Tajima H, et al: Valproic acid inhibits proliferation of HER2-expressing breast cancer cells by inducing cell cycle arrest and apoptosis through Hsp70 acetylation. Int J Oncol 47: 2073-2081, 2015.

124. Kim J, Kundu M, Viollet B and Guan KL: AMPK and mTOR regulate autophagy through direct phosphorylation of Ulk1. Nat Cell Biol 13: 132-141, 2011

125. Fan QW, Cheng C, Hackett C, Feldman M, Houseman BT, Nicolaides T, Haas-Kogan D, James CD, Oakes SA, Debnath J, et al: Akt and autophagy cooperate to promote survival of drug-resistant glioma. Sci Signal 3: ra81, 2010.

126. Fritsch C, Huang A, Chatenay-Rivauday C, Schnell C, Reddy A, Liu M, Kauffmann A, Guthy D, Erdmann D, De Pover A, et al: Characterization of the novel and specific PI3K $\alpha$ inhibitor NVP-BYL719 and development of the patient stratification strategy for clinical trials. Mol Cancer Ther 13: 1117-1129, 2014.

127. Young CD, Arteaga CL and Cook RS: Dual inhibition of Type I and Type III PI3 kinases increases tumor cell apoptosis in HER2+ breast cancers. Breast Cancer Res 17: 148, 2015.

128. Salmaninejad A, Khoramshahi V, Azani A, Soltaninejad E, Aslani S, Zamani MR, Zal M, Nesaei A and Hosseini SM: PD-1 and cancer: Molecular mechanisms and polymorphisms. Immunogenetics 70: 73-86, 2018.

129. Berger KN and Pu JJ: PD-1 pathway and its clinical application: A 20year journey after discovery of the complete human PD-1 gene. Gene 638: 20-25, 2018

130. Kythreotou A, Siddique A, Mauri FA, Bower M and Pinato DJ: PD-L1. J Clin Pathol 71: 189-194, 2018

131. Hartkopf AD, Taran FA, Wallwiener M, Walter CB, Krämer B, Grischke EM and Brucker SY: PD-1 and PD-L1 immune checkpoint blockade to treat breast cancer. Breast Care (Basel) 11: 385-390, 2016

132. Schütz F, Stefanovic S, Mayer L, von Au A, Domschke C and Sohn C: PD-1/PD-L1 pathway in breast cancer. Oncol Res Treat 40: 294-297, 2017.

133. Tsang JY, Au WL, Lo KY, Ni YB, Hlaing T, Hu J, Chan SK, Chan KF, Cheung SY and Tse GM: PD-L1 expression and tumor infiltrating PD-1+ lymphocytes associated with outcome in HER2+ breast cancer patients. Breast Cancer Res Trea 162: 19-30, 2017

134. Hou Y, Nitta H, Wei L, Banks PM, Parwani AV and Li Z: Evaluation of immune reaction and PD-L1 expression using multiplex immunohistochemistry in HER2-positive breast cancer: The association with response to Anti-HER2 neoadjuvant therapy. Clin Breast Cancer 18: e237-e244, 2018.

135. Dirix LY, Takacs I, Jerusalem G, Nikolinakos P, Arkenau HT, Forero-Torres A, Boccia R, Lippman ME, Somer R, Smakal M, et al: Avelumab, an anti-PD-L1 antibody, in patients with locally advanced or metastatic breast cancer: A phase 1b JAVELIN solid tumor study. Breast Cancer Res Treat 167: 671-686, 2018. 